(C) 2015 IEEE. Personal use of this material is permitted. Permission from IEEE must be obtained for all other uses, in any current or future media, including reprinting/republishing this material for advertising or promotional purposes, creating new collective works, for resale or redistribution to servers or lists, or reuse of any copyrighted component of this work in other works. 


\title{
Spherical wind sensor for the atmosphere of Mars
}

\author{
Lukasz Kowalski, M. Teresa Atienza, Sergi Gorreta, Vicente Jiménez, Manuel Domínguez-Pumar, Senior \\ Member, IEEE, Santiago Silvestre, Senior Member, IEEE and Luis M. Castañer, Senior Member, IEEE
}

\begin{abstract}
A novel wind speed and direction sensor designed for the atmosphere of Mars is described. It is based on an spherical shell divided into four triangular sectors according to the central projection of tetrahedron onto the surface of the unit sphere. Each sector is individually controlled to be heated above the ambient temperature independently of the wind velocity and incidence angle. A convection heat rate model of four hot spherical triangles under forced wind has been built with FEM thermal-fluidic simulations. The angular sensitivity of the tetrahedral sphere structure has been determined theoretically and compared with tessellation of the sphere by four biangles. A 9mm diameter prototype has been assembled using 3-D printing of the spherical shell housing in the interior commercial platinum resistors connected to an extension of a custom design printed board. Measurements in Martian-like atmosphere demonstrate sensor responsiveness to the flow in the velocity range $1 \mathrm{~m} / \mathrm{s}$ to $13 \mathrm{~m} / \mathrm{s}$ at $10 \mathrm{mBar} \mathrm{CO}_{2}$ pressure. Numerical modelization of the sensor behavior allows to devise an inverse algorithm to retrieve the wind direction data from the raw measurements of the power delivered to each spherical sector. The functionality of the inverse algorithm is also demonstrated.
\end{abstract}

Index Terms-Mars, wind sensor, tetrahedral sphere, thermal anemometer, low pressure atmosphere

\section{INTRODUCTION}

$\mathbf{T}$ HE measurement of wind direction and speed at the surface of Mars has attracted much attention, especially since the rover Curiosity has landed in the crater Gale on the surface of Red Planet in August $8^{t h}$ 2012. On board of the planetary vehicle/robot was a meteorological station REMS (Remote Environmental Monitoring Station) including several sensors, among them a wind sensor, that has been able since then to send meteorological data to the Earth. The atmosphere of planet Mars is mainly composed of carbon dioxide, has typically a pressure in the range of $6 \mathrm{mBar}$ to $12 \mathrm{mBar}$ and a temperature ranging from $150 \mathrm{~K}$ to $300 \mathrm{~K}$ [1]. Among the several available methods to measure wind speed and direction, the most adequate for this application is based in thermal anemometry. Such low pressures of the atmosphere do not allow proper operation of sensors based on dynamic pressure, as they cannot work in the range of $0.01 \mathrm{~Pa}$ to $1 \mathrm{~Pa}$, that are the expected pressures at a wind speed of $1 \mathrm{~m} / \mathrm{s}$ to $60 \mathrm{~m} / \mathrm{s}$ [2]. Ultrasound based sensors require higher power and do not work at pressure smaller than $15 \mathrm{mBar}$ [3]. Till now the sensors based on laser and Doppler's effect have not yet been sufficiently miniaturized for this application [4], and they need a permanent presence of tracer particles to operate. The heritage of thermal wind sensors for Mars is quite substantial

L. Kowalski and others authors are with the Department of Electronic Engineering (EEL), Universitat Politècnica de Catalunya (UPC), Micro and Nano Technologies research group (MNT), Calle Jordi Girona 1-3, 08032 Barcelona, Spain. e-mail: lukasz.kowalski@upc.edu, webpage: https://www.eel.upc.edu

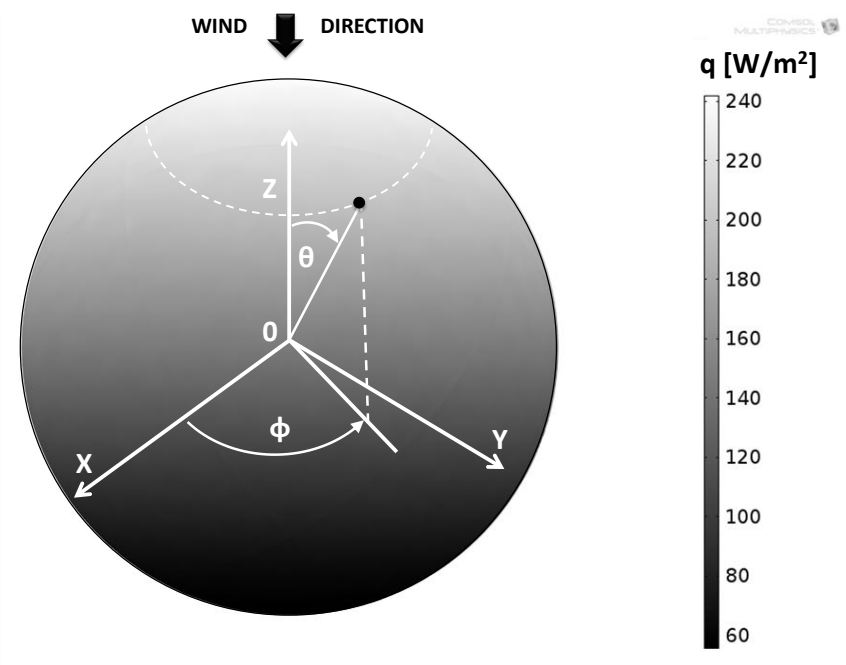

Fig. 1. FEM simulation of the local heat flux $q$ for the hot $(\Delta \mathrm{T}=20 \mathrm{~K})$ sphere $(\mathrm{D}=9 \mathrm{~mm})$ under forced wind $(\mathrm{U}=5 \mathrm{~m} / \mathrm{s})$ coming from $-\vec{z}$ direction in carbon dioxide $\left(\mathrm{CO}_{2}\right)$ Mars-like atmosphere conditions $\left(\mathrm{P}=600 \mathrm{~Pa}\right.$ and $\left.\mathrm{T}_{a m b}=220 \mathrm{~K}\right)$.

as Viking in 1976 incorporated the first that was able to measure wind in the surface of planet Mars [5]-[7] based on thin small cylinders covered by platinum resistors; Pathfinder in 1997 included a cylindrical thermal wind sensor [8]-[11] with heated platinum-iridium wires and MSL rover Curiosity included two booms having silicon chips heated by platinum resistors [12]-[14].

Viking sent wind data for two years while Pathfinder was able to send wind direction data only. Up to date REMS still is operational, although news came after Curiosity rover touched down the Martian ground after 253 days of space cruise, that some of the measurement chips of the two booms were damaged [15]. This has degraded performance of the wind parameters retrieval and thus a limited set of measurements are currently being sent to the Earth. Nevertheless, whirlwinds have been detected [16]. The challenge is difficult as power and mass restrictions are accompanied by the requirements of sufficient interaction with the wind and the need to protect the sensors. The spherical sensor reported here was developed as a UPC (Universitat Politècnica de Catalunya) as contribution to the project MEIGA (Mars Environmental Instrumentation for Ground and Atmosphere) [17]. Actually, the spherical wind sensor described in this paper is a heritage of the REMS Curiosity wind sensor. This is so because the concept is entirely the same as it consists on the heating of resistors above the ambient temperature. In the case of Curiosity the resistors were placed on top of silicon chips and supported by pedestals above the PCB and wire bonded. The number of heated silicon chips in REMS was 4 for every 2-D PCB 
and they were three of those PCB's in the boom. Moreover, there were two booms to overcome the shadow of the camera mast. The spherical wind sensor here have several advantages, being though a direct heritage of REMS: (a) it benefits from the spherical shape of the shell that ensures an axisymmetric and laminar flow around it at the Reynolds numbers expected, (b) To resolve for 3-D wind direction we only need four spherical sectors (and just four resistors) instead of three full PCB's with four resistors each, (c) all resistors are placed inside the spherical shell and hence inherently protected and not vulnerable to wire bonding incidental break-out, (d) the characteristic length of the supporting arm is few times less than the characteristic length of the sensor whereas in REMS the boom size was several times larger than the size of the chip, (e) it benefits from the fact that the whole shell is an active part in contact with the wind flow, (f) the addition of the core resistors allows to minimize the conduction thermal losses from the shell to the supporting structure. Working with only one temperature $\left(T_{h o t}\right)$ together with the geometry isotropy significantly simplifies thermal modelling and reverse algorithm. The purpose of this paper is to describe the idea and first measurements of a spherical miniature wind sensor based also in thermal anemometry that was preliminarily introduced in reference [18].

\section{SPHERICAL WIND SENSOR CONCEPT}

Thermal anemometry wind sensing requires to thermally interact with the environment in such a way that heat is transferred by convection. Convection heat transfer coefficient depends on the dimensionless Reynolds and Prandtl numbers. As the Reynolds number depends on wind speed-pressure product, a measure of the heat transfer coefficient provides a measure of the wind speed. In order to measure the heat transfer coefficient $h$ the thermal conductance to the environment must be monitored

$$
h=\frac{G t h}{A}
$$

where $G t h$ is the thermal conductance and $A$ is the surface coupled to the wind flow.

At the same time, the thermal conductance Gth to the environment can be simply calculated from

$$
G t h=\frac{P w}{\Delta T}
$$

where $P w$ is the power delivered to a hot point setting an overheat temperature $\Delta \mathrm{T}$ above the environment.

Thermal flow sensors have been investigated extensively for a long time. Many of the 2-D thermal flow sensors concept have been realized [19]-[22]. However to our knowledge the 3-D anemometers are built using multiple 2-D anemometers [23]. In three dimensions the wind direction can be measured by placing several 2-D wind sensors in different planes and using an inverse algorithm to retrieve the wind direction from the values of the thermal conductances, as it is the case in the REMS wind sensor [14]. This algorithm can be awkward depending on the specific geometry of the sensor and may not be equally sensitive to all angles. In this case, the

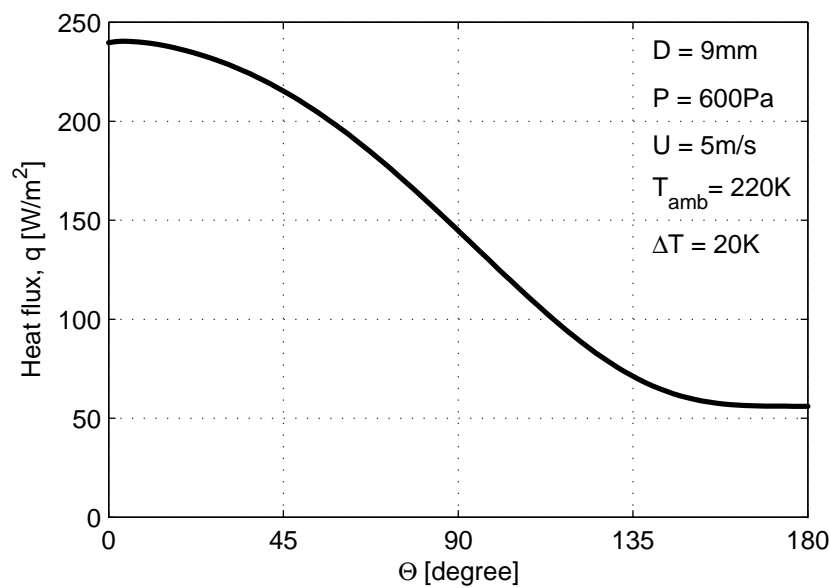

Fig. 2. Local heat flux at any point of a sphere as a function of angle $\Theta$. Raw data extracted from the simulation shown in Fig. 1.

spherical geometry simplifies the problem and is intrinsically 3-D.

Spherical geometry has many advantages that come from the inherent isotropy. Considering a solid sphere subject to a wind flow, the heat convection coefficient will be independent on the wind direction. Furthermore, the sphere can be splitted into several spherical sectors that can be independently controlled. The sectors that are more exposed to the wind will lose heat more than the others and hence a measurement of the difference between sectors will provide the wind direction. The wind magnitude will be given by the heat transfer coefficient of all sectors together.

Let us first consider an sphere as shown in Fig. 1 where a COMSOL simulation of the value of the local convection heat flux $q$ in units of $\left[\mathrm{W} / \mathrm{m}^{2}\right]$ is shown at any point identified by angles $\Phi, \Theta$. This is for the surface of the sphere considering that the flow is impinging the sphere by the uppermost point located at $\Theta=0^{\circ}$ and $\Phi=0^{\circ}$.

In Fig. 1 the diameter of the sphere is $9 \mathrm{~mm}$, the ambient pressure is $600 \mathrm{~Pa}$, the wind temperature is $220 \mathrm{~K}$, the speed of the wind is $5 \mathrm{~m} / \mathrm{s}$ and the temperature of the hot sphere is $240 \mathrm{~K}, 20 \mathrm{~K}$ above the wind temperature. As can be observed from Fig. 1, there is revolution symmetry around the Z-axis and in the most exposed parts to the wind is where the power delivered is greater to maintain the overheat at any point at the same value. This is clearly seen in Fig. 2 where the local heat flux $q$ is plotted as a function of angle $\Theta$ for any value of angle $\Phi$.

Comprehensive details of how the multi-physics simulations were performed will be addressed separately in a forthcoming publication. These simulation results are compared to the thermal conductance values calculated from empirical approximation of Nusselt number proposed by Feng \& Michaelidas [24], as shown in Fig. 3.

The thermal conductance of the sphere is independent of the wind direction due to the symmetry. Let us now describe how the wind direction can also be measured using as baseline the spherical geometry just described. This is achieved by breaking the sphere surface into spherical sectors physically 


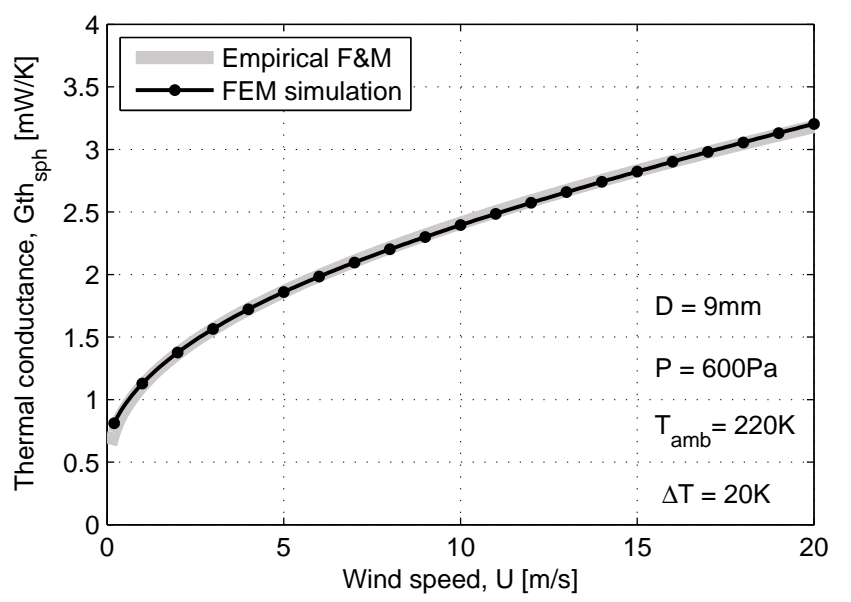

Fig. 3. Thermal conductance for the whole sphere as a function of the wind speed. Empirical model based on Feng \& Michaelides (F\&M) compared to FEM simulation results.

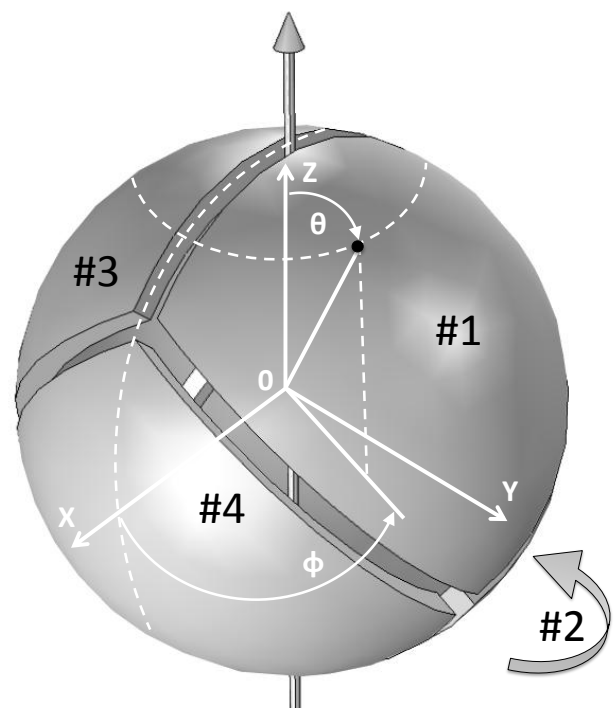

Fig. 4. Physical model of spherical shell division based on tetrahedron geometry into separated from each other four triangular sectors. Sectors: \#1, $\# 3$, \#4 can be seen from the picture, whereas sector \#2 is in the rear of the plot. Sectors orientation according to the Cartesian coordinate system are presented as well as theoretical wind incidence point (represented by black dot) described with polar angles $\Theta$ and $\Phi$.

separated as depicted in Fig. 4 where an example of four independent sectors is shown.

In this work, four sectors were used to solve for the two angles of the wind direction $(\Theta, \Phi)$, extending the results we had using just two hemispherical sectors that allow only to solve for one angle as reported in [18]. We have considered not using more than four sectors for practical fabrication issues and because the angle sensitivity achieved with four sectors - as will be shown below - is currently above the typical requirements in Mars missions. The four sectors geometry shown in Fig. 4 are spherical triangles having the centers located at points with coordinates for sector $\# 1$ at $\left(\Theta=54.74^{\circ}\right.$ and $\left.\Phi=90^{\circ}\right)$, for sector $\# 2$ at $\left(\Theta=125.56^{\circ}\right.$ and $\left.\Phi=180^{\circ}\right)$, for sector \#3 at $\left(\Theta=54.74^{\circ}\right.$ and $\left.\Phi=270^{\circ}\right)$ and for sector \#4 at $\left(\Theta=125.56^{\circ}\right.$ and $\left.\Phi=360^{\circ}\right)$. This particular geometry was

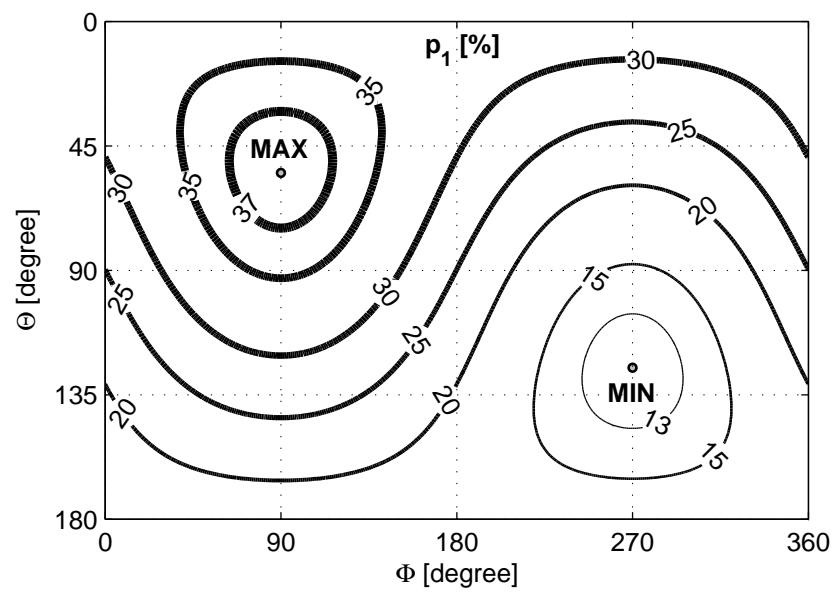

Fig. 5. Percentage of the total sphere convection heat rate transferred to the ambient by the triangular sector \#1 as function of wind direction angles $(\Theta, \Phi)$. Values produced from the simulation data presented in Fig. 2 according to the sector \#1 orientation shown in Fig. 4.

obtained by expansion of an exploded tetrahedron over a spherical shell.

The local heat flux integrated along the surface of each sector gives the convection heat rate $\dot{Q}_{i}$

$$
\dot{Q}_{i}=\oint_{A_{i}} q \cdot d A
$$

where $q$ is a local heat flux along the surface $A$ of the sector $i$. The total heat rate for whole sphere $\dot{Q}_{s p h}$ is given by

$$
\dot{Q}_{s p h}=\dot{Q}_{1}+\dot{Q}_{2}+\dot{Q}_{3}+\dot{Q}_{4}
$$

The percentage heat share $p_{i}$ which corresponds to a given sector $i$ is

$$
p_{i}=\dot{Q}_{i} / \dot{Q}_{s p h} \cdot 100 \%
$$

The values of $p_{i}$ are shown in Fig. 5 where the convection heat rate of sector \#1 is plotted in percentage of the global heat rate of the whole sphere as a function of the direction angles of the wind vector $(\Theta, \Phi)$.

As can be seen, the maximum percentage of the heat rate value is $37.84 \%$ of the total sphere and it is achieved when the wind comes from the direction $\left(\Theta=54.74^{\circ}\right.$ and $\left.\Phi=90^{\circ}\right)$ directly into the center of the sector \#1 - and has a minimum of $12.15 \%$ when the wind comes from the direction $\left(\Theta=125.26^{\circ}\right.$ and $\Phi=270^{\circ}$ ) - the opposite direction to the sector's central point where the other three sectors: \#2, \#3, \#4 join together. The plot shown in Fig. 5 for sector \#1 is numerically the same for the other three sectors provided a proper shift in the axes for the angles $\Phi$ and $\Theta$ is performed. They are not plotted here for simplicity.

Now let's consider a case when we deal with a wind within horizontal plane, where $\Theta=90^{\circ}$, and full rotation along $\Phi$ angle is considered. In that case data for sector \#1 can be easily extracted from Fig. 5. This has been done also for the other three sectors and the results are shown in Fig. 6. The curves resemble shifted sinusoidal functions. 


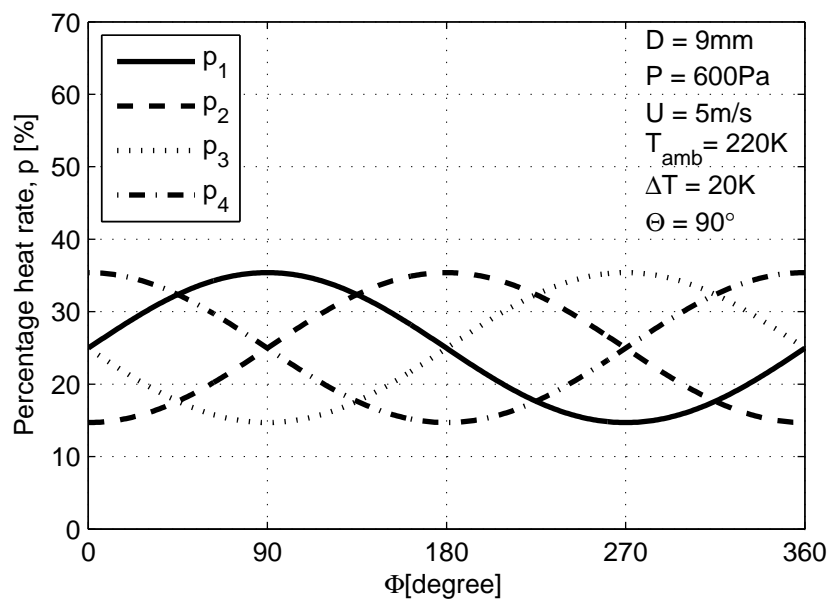

Fig. 6. Percentage of the total sphere convection heat rate which goes to the each of the four sectors as a function of wind angle $\Phi$, for $\Theta=90^{\circ}$.

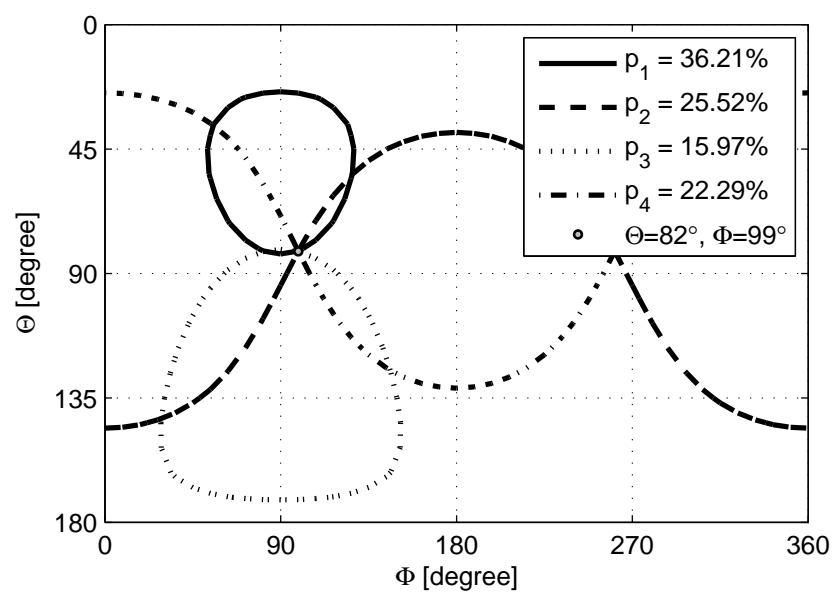

Fig. 7. Wind direction angles $(\Theta, \Phi)$ as a cross point of iso-percentage convection heat rate curves for all sectors.

The algorithm to resolve the wind direction from the measurement of the thermal conductances of the four sectors is described in Fig. 7, where an example is illustrated for wind direction: $\Theta=82^{\circ}, \Phi=99^{\circ}$. In this figure the superposition of the plots of percentage of the heat rate of the four sectors is shown. As can be seen, there is only a point where all four plots intersect. The coordinates of this point provides the value of the angles of the wind direction and corresponds to a power split among the four sectors as follows: $36.21 \%$ goes to sector \#1, 25.52\% goes to sector \#2, 15.97\% goes to sector \#3 and $22.29 \%$ goes to sector \#4.

In order to find out theoretically the sensor sensitivity to the change of incidence angle we have performed the numerical calculation of the angle derivatives of the convection heat rate for the four sectors that allows to calculate angle sensitivities, $S_{\Theta}$ and $S_{\Phi}$

$$
\left\{\begin{array}{l}
S_{\Theta}=\frac{\left\|\frac{\partial \vec{Q}}{\partial \Theta}\right\|_{2}}{\dot{Q}_{s p h}} \cdot 100 \% \\
S_{\Phi}=\frac{\left\|\frac{\partial \vec{Q}}{\partial \Phi}\right\|_{2}}{\dot{Q}_{s p h}} \cdot 100 \%
\end{array}\right.
$$

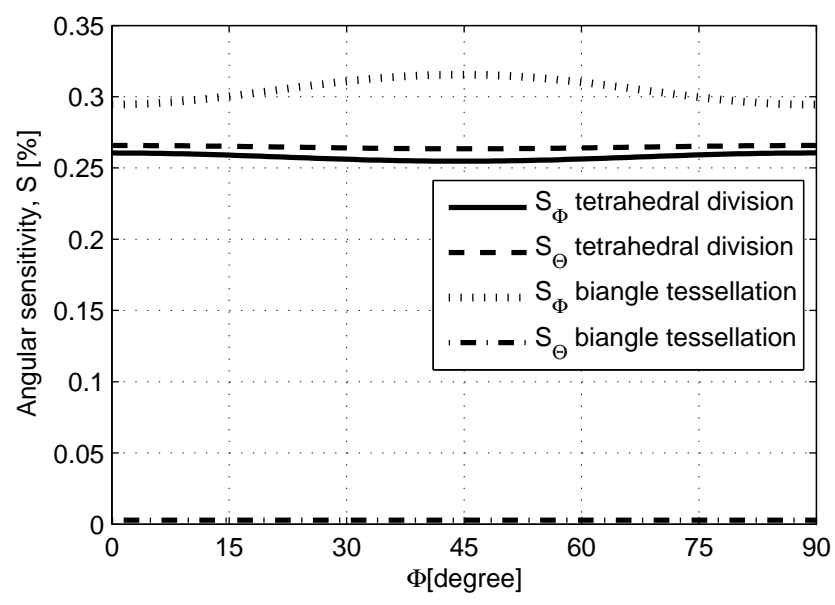

Fig. 8. Angular sensitivity plots for two type of spherical geometries division into four equal sectors. Traditional biangle tessellation has been contrasted with tetrahedral division. $S_{\Theta}$ and $S_{\Phi}$ for both geometries and wind speed of $7 \mathrm{~m} / \mathrm{s}$ can be observed for $\Phi$ rotation range $0^{\circ}$ to $90^{\circ}$ and for $\Theta=90^{\circ}$.

where $\left\|\frac{\partial \vec{Q}}{\partial \Theta}\right\|_{2}$ and $\left\|\frac{\partial \vec{Q}}{\partial \Phi}\right\|_{2}$ are the norms of the heat vectors derivative given by,

$$
\left\{\begin{array}{l}
\left\|\frac{\partial \vec{Q}}{\partial \Theta}\right\|_{2}=\sqrt{\sum_{i=1}^{4}\left(\frac{\Delta \dot{Q}_{i}}{\Delta \Theta}\right)^{2}} \\
\left\|\frac{\partial \vec{Q}}{\partial \Phi}\right\|_{2}=\sqrt{\sum_{i=1}^{4}\left(\frac{\Delta \dot{Q}_{i}}{\Delta \Phi}\right)^{2}}
\end{array}\right.
$$

The angular sensitivities are shown in Fig. 8. The curves have been plotted for $\Theta=90^{\circ}$ and for values of $\Phi$ ranging from $0^{\circ}$ to $90^{\circ}$ which covers the entire rotation due to the symmetry. The results show that both sensitivities $S_{\Theta}$ and $S_{\Phi}$ are very similar and very homogeneous for all rotation angles. In this example a $1^{\circ}$ change in the wind direction angle results in relocation of $0.25 \%$ of total heat rate delivered to the whole sphere among the sectors, whereas the total heat rate remains constant. This is compared in the same figure with similar calculations for an sphere classical biangular division into four equal sectors. As can be seen in this case, the sensitivity is larger for angle $\Phi$ and not homogenous but at the same time sensitivity for $\Theta$ is very small. Those results motivated our decision to work with four sectors divided into triangular sectors as shown in Fig. 4.

Fig. 9 shows the value of the minimum sensitivity $S_{\min }$, defined as the smallest of $S_{\Theta}$ and $S_{\Phi}$, as a function of the wind speed for few ambient temperatures. Sensitivity of $0.2 \%$ and higher for the velocity in the range $3-20 \mathrm{~m} / \mathrm{s}$ are found, whereas it decreases for lower wind speed values.

\section{SPHERICAL WIND SENSOR FABRICATION}

The fabrication of the wind sensor prototype includes the fabrication of the shell, the printed circuit board design, the integration and assembly of the pieces along with the platinum resistors. This is schematically drawn in Fig. 10. The spherical sectors are shown in Fig. 10(a) where software models of the outer and inner faces of the shell are depicted as well as a 3-D picture of the assembly of the four sectors Fig. 10(d). 


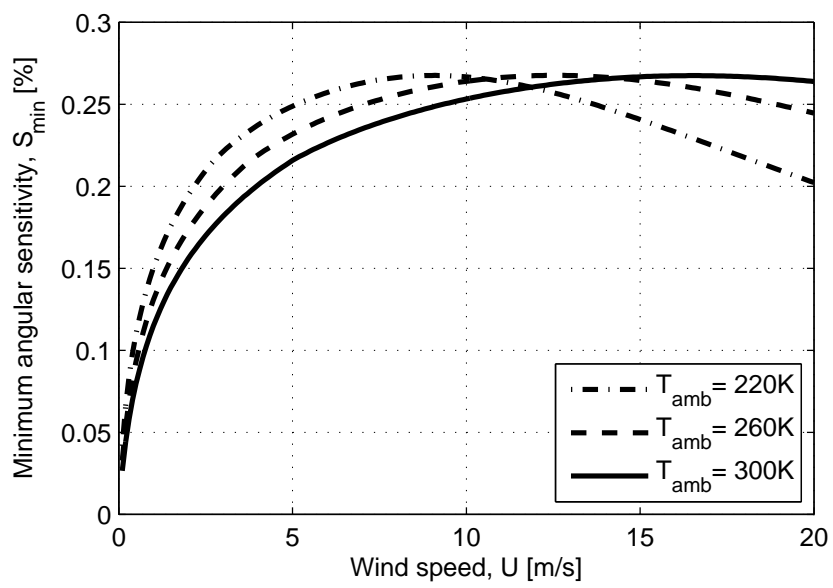

Fig. 9. Minimum angular sensitivity analysis of tetrahedral sphere structure working in $\mathrm{CO}_{2} 600 \mathrm{~Pa}$ atmosphere as a function of wind speed and for different ambient temperatures scenario at constant overheat $20^{\circ} \mathrm{C}$.
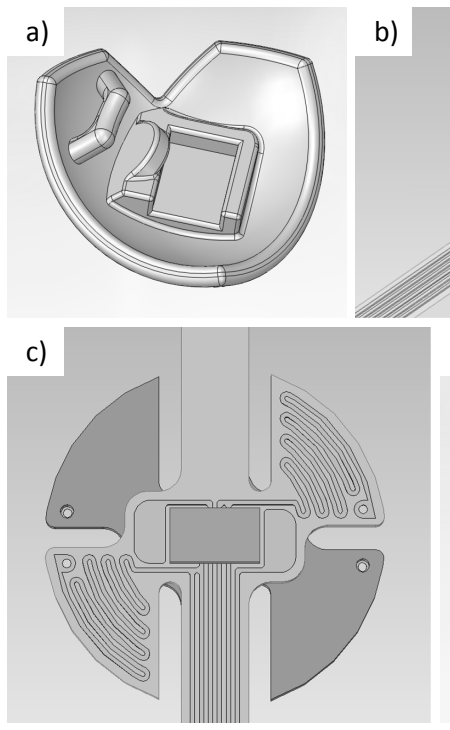

b)

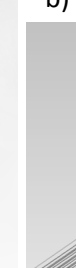

d)

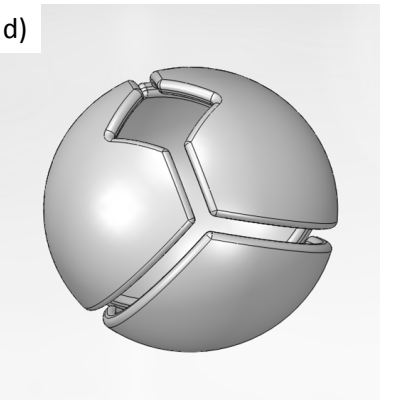

Fig. 10. Models of the spherical wind sensor components, (a) triangular sector with its interior heat resistor mold, (b) PCB with electrical layout and central core resistor, (c) union of the two PCB's were mechanical support and electrical wiring are provided for each sector, (d) four triangular sectors assembly.

All four sectors are interchangeable with each other and are modified spherical triangles to allow the PCB to pass inside. As can be seen, the inner face of the shell includes protrusions to accommodate the platinum resistor and to hold the PCB. The part of the PCB that passes inside the shell is shown in Fig. 10(b), where the copper layout is clearly seen and also the bonding PAD's. The connection between the PAD's and the main frame of the PCB is serpentine shaped to increase the thermal resistance of this path. Moreover, there are also shown the connecting PAD's of a core resistor that is placed on the PCB itself and that has the function to keep the temperature of the PCB at the same temperature as the spherical shell. The printed circuit board was designed in a complementary way that once rotated 180 degree become compatible with
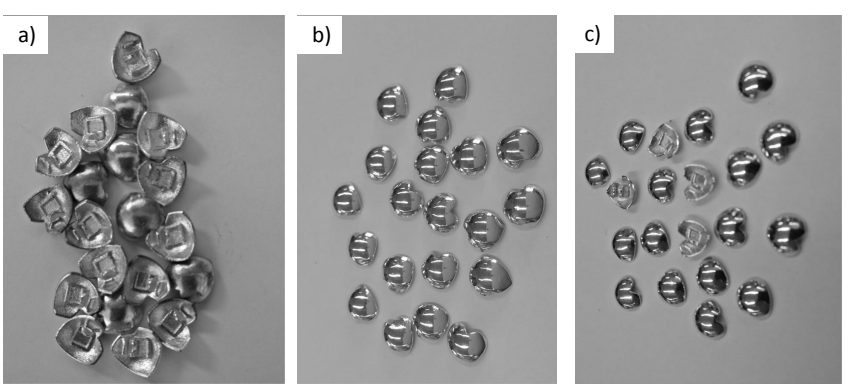

Fig. 11. Pictures of wind sensor triangular sectors, (a) made out of Silver Sterling material (alloy $\mathrm{Au} 92.5 \%, \mathrm{Cu} 7.5 \%$ ) in a stereo-litography 3-D printing commercial process, (b) after tumbler polishing operations with progressive grit, (c) after gold thin film (100nm) deposition on the outer surface. Every sector is $0.3 \mathrm{~g}$ in weight approximately.

a second PCB and creates the structure to give mechanical support and electrical wiring for the four sectors, as shown in Fig. 10(c). The PCB arm-tie has a section of $1.6 \mathrm{~mm}$ which is much smaller compared to the characteristic length of the sphere itself ( $9 \mathrm{~mm}$ in diameter). That was to provide laminar flow around the arm that would not interact largely with the measurement planes.

These specific design solution was selected on purpose to, on the one hand, separate thermally each sector from the others to avoid heat cross effects and, on the other hand, to suppress the heat conduction losses defined by

$$
\dot{Q}_{\text {cond }}=\frac{T_{i}-T_{P C B}}{R t h}
$$

where $T_{i}$ is the hot point temperature of $\# \mathrm{i}$ sector, $T_{P C B}$ is the temperature of the central core region of the PCB and $R t h$ is a thermal resistance of the path from the sector to the PCB support.

The heat conduction thermal losses $\dot{Q}_{\text {cond }}$ are canceled when $T_{i}$ and $T_{P C B}$ have the same value $\left(T_{h o t}\right)$. More details are given elsewhere [18].

For the shell fabrication, a stereo-lithography 3-D printing process of silver $92.5 \%$ and copper $7.5 \%$ in weight, was chosen. This material provides a very high thermal conductivity and allows to achieve an homogeneous temperature distribution. The thickness of the shell is $0.5 \mathrm{~mm}$ and the separation between sectors is also $0.5 \mathrm{~mm}$. Once the sectors were manufactured they were polished by using a tumbler with plastic pellets progressively going from rough to smooth until mirror finish was achieved. After that, a 100nm thin gold layer was sputtered on the outside. The process steps can be seen in Fig. 11 from manufactured (a) to polished (b) and finished (c). The gold coating and the polish process are meant to reduce radiation heat losses by lowering the surface emissivity factor as much as possible [25].

In order to control hot point temperature a thermistor whose resistance depends on temperature has been used as it also can be used as a heating resistor. Any thermistor with linear dependence of its electrical resistivity with the temperature is suitable such as tungsten $\left(\alpha_{W}=0.0044\right)$, platinum $\left(\alpha_{P t}=0.00392\right)$ or copper $\left(\alpha_{C u}=0.00386\right)$. We have chosen platinum for this work as they are commercially available with wide variety of 


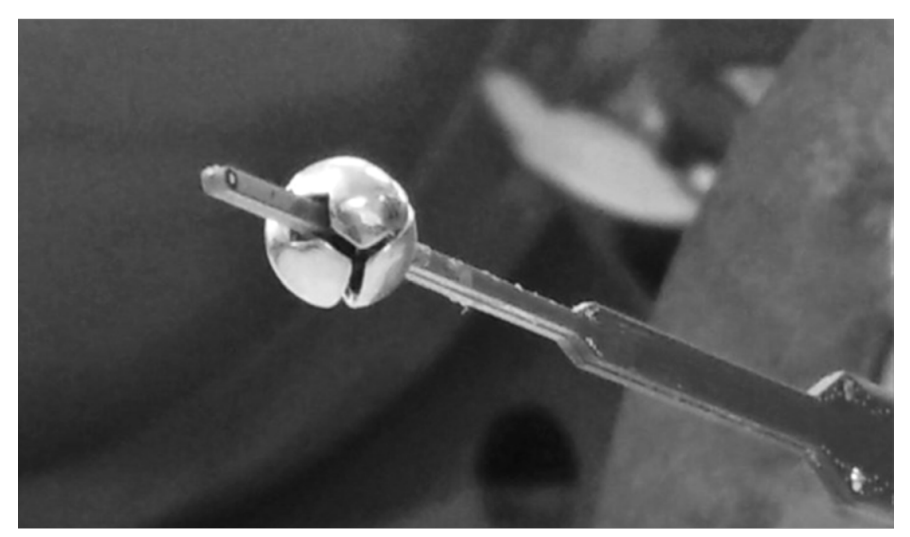

Fig. 12. Picture of the spherical 'tetrahedral' wind sensor prototype, taken after measurements in a Mars-like conditions.

shapes, sizes and values. Surface mounted OMEGA Pt100 resistors having temperature coefficient $\alpha_{T}=0.00385$ have been selected; SMD0603 for the spherical sectors and SMD0805 for the PCB.

A picture of the prototype assembled is shown in Fig. 12 where the outer face of the spherical sensors and the PCB passing trough the inside volume can be seen. The six platinum resistors, one per each sector, and two for the PCB are hidden inside.

\section{WIND SPEED AND DIRECTION MEASUREMENTS}

A substantial set of measurements were performed in the University of Aarhus Mars wind tunnel facility AWTSI, were low pressure and low temperature can be independently set [26]. The measurement conditions are described in Table I.

TABLE I

MEASUREMENT CONDITIONS

\begin{tabular}{ll}
\hline Atmosphere & $\mathrm{CO}_{2}$ \\
\hline \hline $\mathrm{P}[\mathrm{mBar}]$ & 10 \\
$\mathrm{~T}_{a m b}\left[{ }^{\circ} \mathrm{C}\right]$ & $0,-8,-18$ \\
$\Delta \mathrm{T}\left[{ }^{\circ} \mathrm{C}\right]$ & 25,40 \\
$\mathrm{U}[\mathrm{m} / \mathrm{s}]$ & $1,2,4,7,10,13$ \\
$\Theta[$ degree $]$ & 90 \\
$\Phi[$ degree $]$ & $45,75,90,105,135$ \\
\hline
\end{tabular}

Temperature and pressure has been set at the begging of each experiment. Minor drifts, though, have been observed during the experiment. In the case of the temperature, the fluctuation is below $\pm 1^{\circ} \mathrm{C}$ Regarding pressure, a small vacuum leak generates a progressive drift (for the duration of the experiment) of $2 \mathrm{mBar}$.

Every hot element works at constant temperature (CTA: constant temperature anemometry). Thermal control circuit implements a constant temperature operation mode using a constant voltage feedback loop [27]. Hence, the temperature of the hot elements in closed loop is independent of ambient temperature. As wind sensor belongs to meteorological station the air temperature is monitored independently and its value is used to infere the value of the thermal conductance to the ambient. Additionally, since there are large temperature swings in Mars atmosphere, a second control loop adapts the value

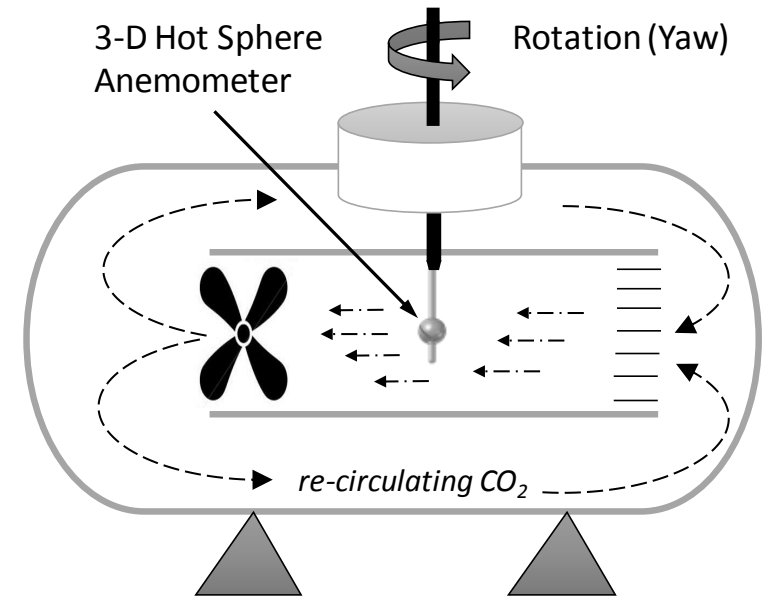

Fig. 15. Orientation of the 3-D hot sphere anemometer inside re-circulating carbon dioxide wind tunnel chamber.

of the overheat temperature over the air temperature ensuring a sufficient difference.

The sensor head was placed inside the chamber and could be rotated in a horizontal plane for 90 degrees, see Fig. 15 . The typical set of measurements performed, was for wind speed changing every 200s. For each wind speed the rotation angle $\Phi$ was varied from $45^{\circ}$ to $135^{\circ}$ with intermediate values: $75^{\circ}, 90^{\circ}, 105^{\circ}$ an then back to $45^{\circ}$ before flow velocity was changed. Each measurement sequence for wind velocity and angle variation has been performed considering two overheat conditions for hot element: $25^{\circ} \mathrm{C}$ and $40^{\circ} \mathrm{C}$. System can work either with a low o high overheat. Nevertheless, by having a higher overheat the convection heat flow is also higher as it depends linearly with the overheat temperature. What is more, by providing higher overheat value CTA system is less affected by ambient temperature variation on Mars and by the temperature fluctuation that happened inside measurement chamber during the tests.

An example set of measurement for $\mathrm{CO}_{2}$ at $P \approx 10 \mathrm{mbar}$ and $T_{a m b} \approx-18^{\circ} \mathrm{C}$ and overheat $\Delta T=40^{\circ} \mathrm{C}$ is shown in Fig. 13, where values of the average power for each sector calculated during the corresponding slot of velocity and flow direction are plotted.

Fig. 13(a) shows for each velocity the raw data of the power delivered to each sector as a function of the $\Phi$ angle from $45^{\circ}$ to $135^{\circ}$. As can be seen, the sensor has sufficient sensitivity to the incidence angle of the wind as the four different sectors require different amount of power to keep the same overheat temperature above the ambient.

Precisely, this difference is what is used to calculate the value of the incidence angle of the wind thereby providing direction sensitivity. This is clearly seen for the plot Fig. 13(b) or the same wind velocity $U=7 \mathrm{~m} / \mathrm{s}$ and consecutive wind angles: $45^{\circ}, 75^{\circ}, 90^{\circ}, 105^{\circ}, 135^{\circ}$ and back to $45^{\circ}$, whereas as predicted buy the isotropy of the sphere the sum of the sector's power is independent of the wind incidence angle thereby providing with the wind speed value.

For each velocity the measurement starts and finishes with the same wind direction angle $45^{\circ}$ so it could be expected 
a)
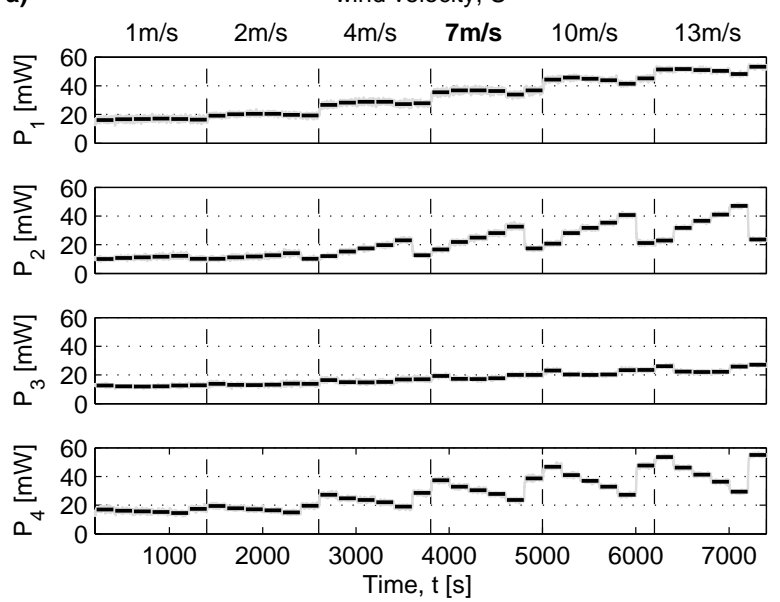

b)

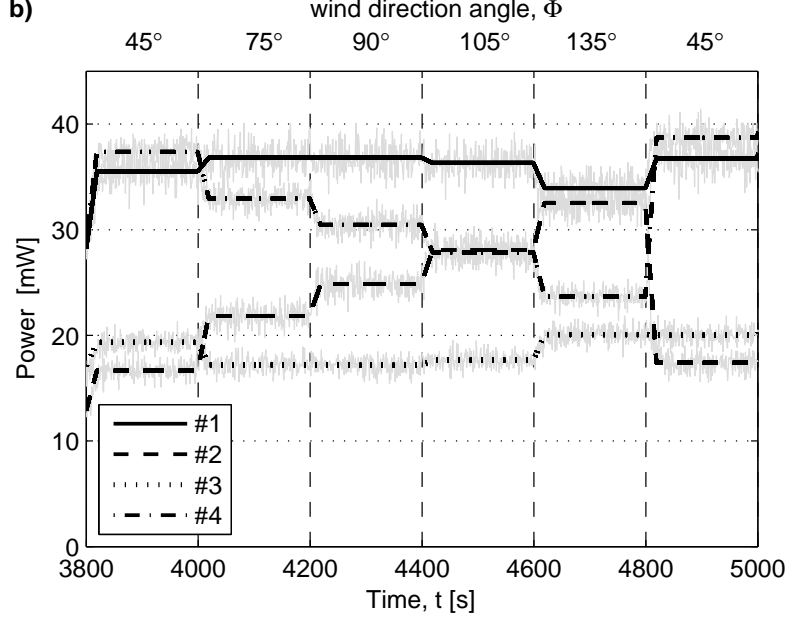

Fig. 13. Power delivered to the four spherical sectors (\#1, \#2,\#3, \#4), (a) for different wind velocities, where for each velocity $(U)$, the specific wind direction angle $(\Phi)$ sequence $\left[45^{\circ}, 75^{\circ}, 90^{\circ}, 105^{\circ}, 135^{\circ}, 45^{\circ}\right.$ ] was tested, (b) zoom on the section where the wind velocity was $7 \mathrm{~m} / \mathrm{s}$ and only wind direction sequence is on display. Measurements performed in $\mathrm{CO}_{2}$ atmosphere at $P \approx 10 \mathrm{mBar}$ and $T_{a m b} \approx-18^{\circ} \mathrm{C}$ conditions, for $\Theta=90^{\circ}$ and overheat $\Delta \mathrm{T}=40^{\circ} \mathrm{C}$.
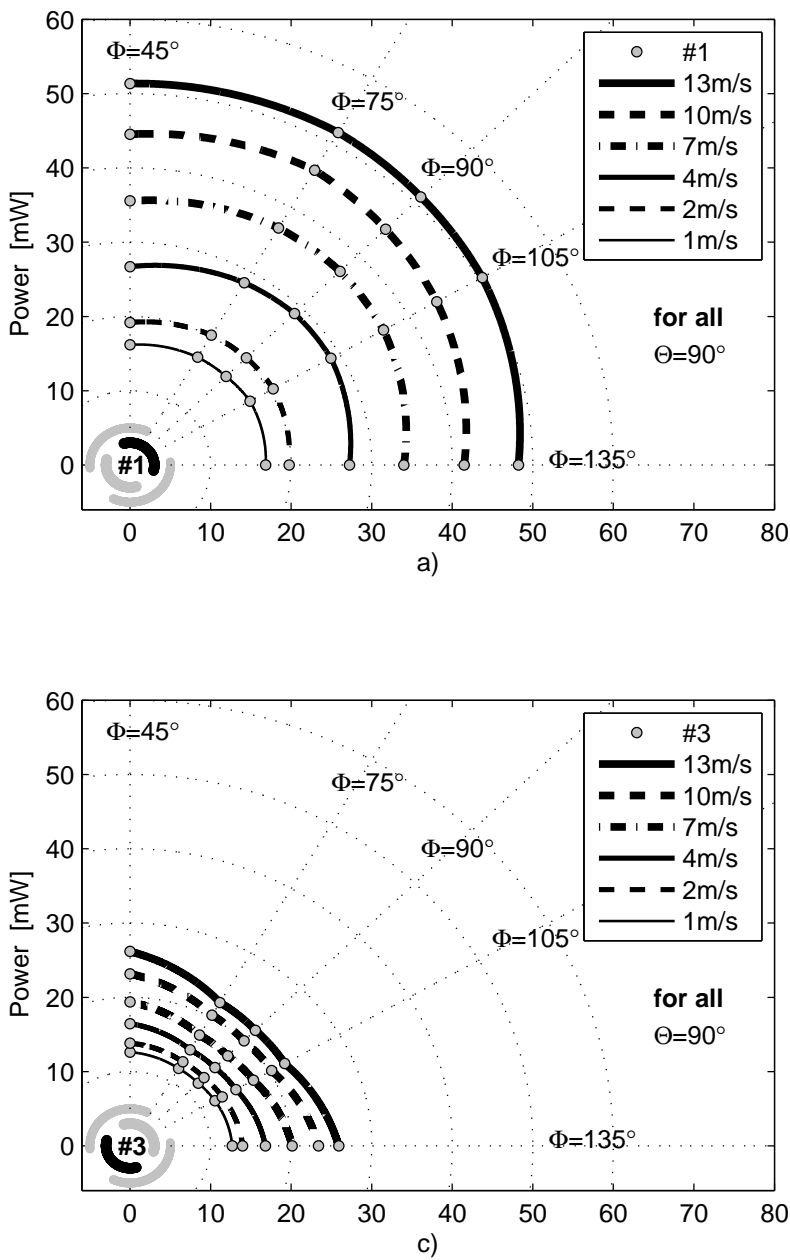
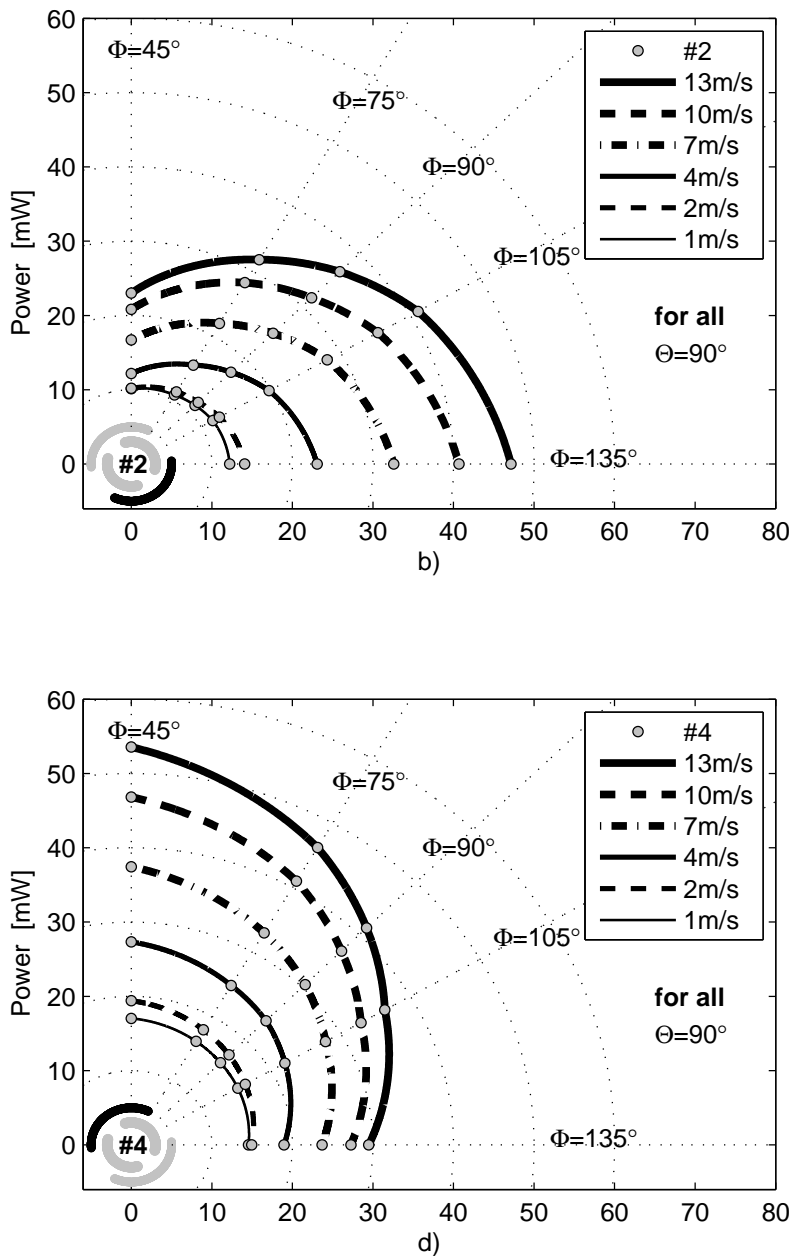

Fig. 14. Polar plot of the average power delivered to the four sectors: (a) sector \#1, (b) sector \#2, (c) sector \#3, (d) sector \#4 as function of the wind speed and wind direction angle $\Phi$, for $\Theta=90^{\circ}$. 


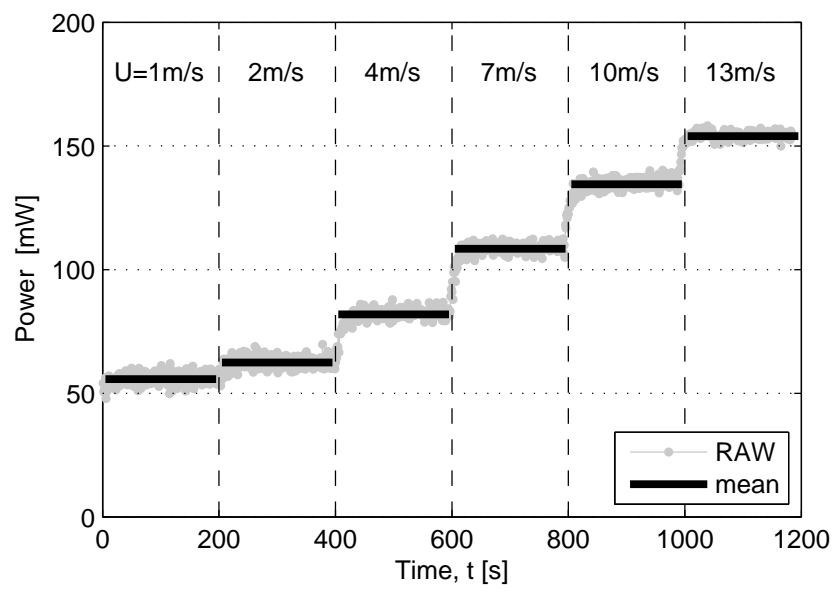

Fig. 16. Raw measurements of total power delivered to the sphere for different wind speed blowing from direction $\Phi=45^{\circ}$ and $\Theta=90^{\circ}$ together with mean power value calculated for each wind direction slot.

that the power readout for the same angle would be the same. However, in Fig. 13(b) it can be seen that the power for all sectors increases around $2 \%$. We attribute this change to the $4 \%$ rise in the $P \cdot U$ product as the chamber pressure changed during experiment from $11.3 \mathrm{mBar}$ to $11.8 \mathrm{mBar}$.

Fig. 14 shows the measured data (filtered from the raw data) of the power delivered to every spherical sector as a function of the angle of incidence and of the magnitude of wind speed.

As the experimental set up did not allow to change the angle $\Theta$, which was fixed to $90^{\circ}$, the angle that we changed was $\Phi$ from $45^{\circ}$ to $135^{\circ}$. We were satisfied with this range since the tetrahedral division of the sphere shows symmetry in the power readout every $90^{\circ}$ as can be seen in Fig. 6 .

Results obtained for the sector \#2 are shown in Fig. 14(b). This sector has an orientation relative to the tunnel axis as shown in the inset. This is a polar plot where the radius is the value of the power. Each line corresponds to different wind speeds and the data points corresponds to the various angles explored. For example, sector \#2, under wind speed of $13 \mathrm{~m} / \mathrm{s}$ from direction $\Theta=90^{\circ}$ and $\Phi=45^{\circ}$, required a power of $23 \mathrm{~mW}$ to keep an overheat temperature of $40^{\circ} \mathrm{C}$ above the ambient. As can be seen, due to the position of this particular sector in the sphere shell, the power required increases as the angle increases. Similar information is shown for the other three sectors in figures Fig. 14(a), (c) and (d). This plot provide an interesting overview of the sensor behavior especially if we look at the pair of sectors with opposite location around the PCB. From pair of sectors \#1 and \#3 we see that when the wind blows into sector \#1 it requires maximum power, whereas the opposite sector \#3 demands minimum power. On the other pair of the sectors \#2 and \#4 we can observe that when the wind incidence angle is changing toward the center of the sector \#2 its power increases whilst the same angular change results in changing wind incidence angle away from the sector \#4 its power decreases. What also stands out from Fig. 14 is that power plots for different velocities but for the same angles do not cross each other.

In Fig. 16 the value of the sum of the power delivered to

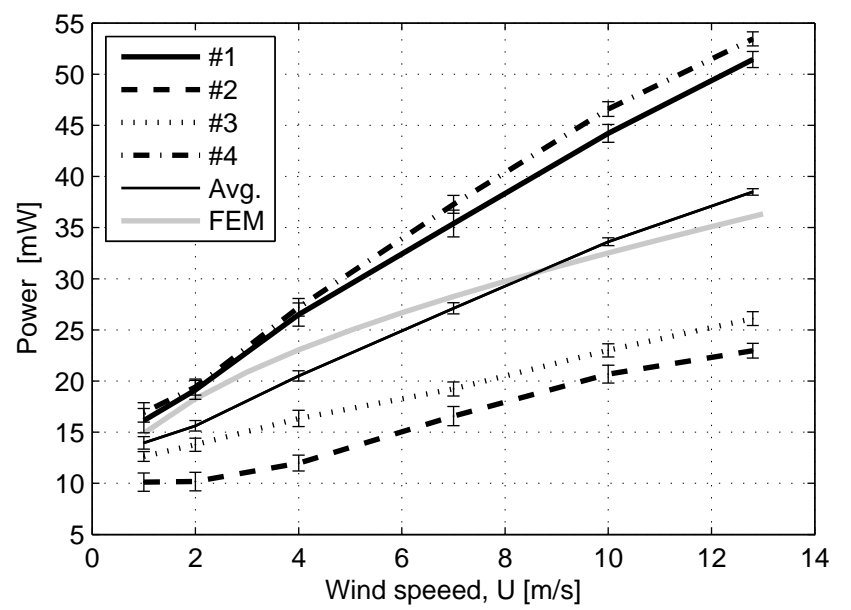

Fig. 17. Mean power delivered to each sector and average sector power demand as a function of the wind speed for wind direction $\Theta=90^{\circ}$ and $\Phi=45^{\circ}$. Data selected from the Fig. 13, whereas FEM curve (for average sector power) was added from multi-physic numerical simulation for the same ambient parameters. $P_{\text {Avg. }}=\left(P_{1}+P_{2}+P_{3}+P_{4}\right) / 4=P_{S p h} / 4$

the four sectors is plotted as a function of time for constant wind direction angle $\Phi=45^{\circ}$ and $\Theta=45^{\circ}$. The wind speed corresponding to every time slot is also shown. As can be see, velocities of wind ranging from $1 \mathrm{~m} / \mathrm{s}$ up to $13 \mathrm{~m} / \mathrm{s}$ were produced at the ambient pressure of about $10 \mathrm{mBar}$ and at local $\mathrm{CO}_{2}$ temperature of $-18^{\circ} \mathrm{C}$. A custom acquisition board that maintains the target temperature in each sector has been developed and used for the measurement. Details of the electronic circuit used and the measurement strategy will be reported separately.

The raw data shown in Fig. 16 demonstrate sensitivity to the wind speed and sufficient stability of the measurement taking into account that the ambient temperature and pressure were set at the beginning of each experiment, and some temperature and pressure drifts were recorded during measurements. As the wind velocity grows, increase in the global sphere power demand was registered.

Fig. 17 is an example of power demand for a particular sphere orientation relative to the wind direction $\Theta=90^{\circ}$ and $\Phi=45^{\circ}$. The mean power delivered to each sector of the sphere is plotted against the wind velocity value as well as average sector power for all sectors. In the same figure numerical simulation results obtained by FEM were added. This multiphysics simulation was performed using COMSOL for the same ambient conditions as inside the wind tunnel chamber during the experiment. What stands out from this figure is that the power convection curve from simulation is in good agreement with experimental data obtained in measurements. Another interesting thing observed in Fig. 17

is that for all wind speeds the power drained by sectors \#1 and \#4 is similar and much higher than power delivered to the pair of sectors \#2 and \#3 (also quite the same) and this is what has been predicted for a wind direction within horizontal plane for angle $\Phi=45^{\circ}$ in Fig. 6 .

The algorithm to find out the wind direction from the measurement of the power of the four sectors is graphically 


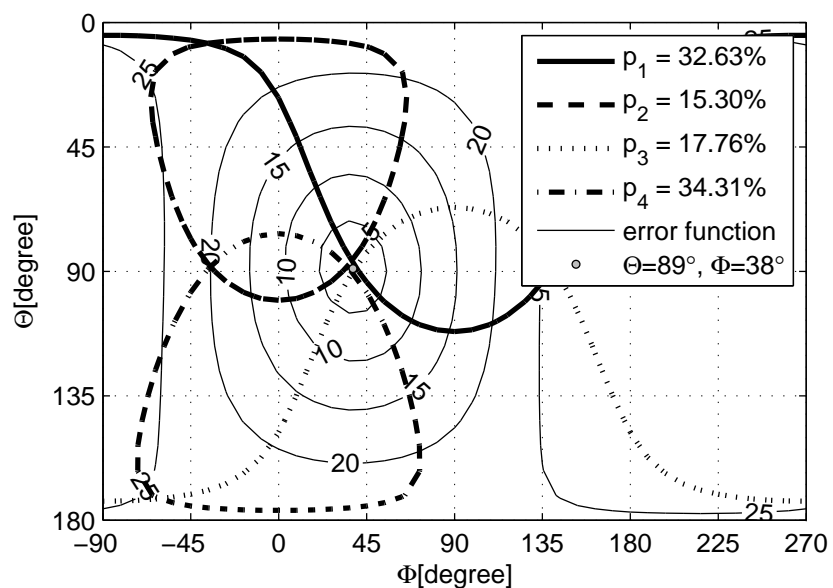

Fig. 18. Wind incidence angle discrimination from real measurement of the power delivered to four triangular sectors. Iso-percentage curves were superposed in order appoint cross section zone. Final wind incidence angle point $(\Theta, \Phi)$ was determined by minimizing en error function.

illustrated in Fig. 18. The same algorithm has been applied for data interval from 3800 s to 4000 s from the measurement set shown in Fig. 13(b). Thus, percentage share of the power which corresponds to each sector has been calculated and shown in Table II.

TABLE II

PERCENTAGE POWER SHARE

\begin{tabular}{|c|c|c|}
\hline sector & Power [mW] & percentage [\%] \\
\hline \hline$\# 1$ & 35.40 & 32.63 \\
$\# 2$ & 16.56 & 15.30 \\
$\# 3$ & 19.23 & 17.75 \\
$\# 4$ & 32.27 & 34.31 \\
Sph & 108.46 & 100.0 \\
\hline
\end{tabular}

As we took the data values for the four sectors corresponding to an experimental incidence angle set for: $\Theta=90^{\circ}$ and $\Phi=45^{\circ}$ and wind velocity $U=7 \mathrm{~m} / \mathrm{s}$, we performed numerical simulations for the same wind atmospheric conditions in order to reproduce the percentage plots, similarly to what we have done in Fig. 7 for every incidence angle. To verify that we can use an algorithm to retrieve the incidence angle from the measurements we have taken the values of percentage power share: $\mathrm{p}_{1}, \mathrm{p}_{2}, \mathrm{p}_{3}$, and $\mathrm{p}_{4}$ from Table II, then have plotted isopower plots for every combination of the incidence angles. In consequence, we have obtained the four plots shown in bold line in Fig. 18.

As it can be seen, there is not a single intersection point for all curves as theoretically predicted in the example shown in Fig. 7, but several intersection points for each pair of curves that are close to each other. This can be associated to different issues. Of course, fabrication and assembly tolerances may lead to non-totally ideal geometry. Moreover, the theoretical algorithm does not contemplate the protruding arm-tie, end even if the wind pass smoothly the arm-tie without as much disturbance (dipper laminar flow than for the sphere due to down-scaled its characteristic size) it may gently affect local heat flux. This is something requiring further investigation. There could also be minor temperature gradients within the shell although they are very small due to the high thermal conductivity of the silver shell $(430 \mathrm{~W} / \mathrm{mK})$. The predicted temperature non-uniformity is below a $0.2 \%$. Another reason may be that the experimental conditions may have drifted from the initial settings in the experimental chamber. Some radiation losses, although very low and not considered in this simplified heat model, may have been produced some power offset. Finally, measurement errors due to long wiring set-up could sum in wind angle discrimination plot.

Therefore, in order to get the angle of incidence of the wind more precisely, we calculated the value of the mean square error between the measured values and all possible obtained from ideal FEM simulations as follows,

$$
\begin{gathered}
(\hat{\Theta}, \hat{\Phi})=\underset{\Theta \in[0, \Pi]}{\operatorname{Arg} \text { Min }}\left\|\vec{p}_{m}-\vec{p}_{s}(\Theta, \Phi)\right\|_{2} \\
\Phi \in[0,2 \Pi)
\end{gathered}
$$

where $\vec{p}_{m}$ is the vector of the percentage value of the heat rate for the measurement at unknown 3-D wind angle,

$$
\vec{p}_{m}=\left(\begin{array}{c}
p_{1} \\
p_{2} \\
p_{2} \\
p_{4}
\end{array}\right)
$$

and $\vec{p}_{s}$ is the vector of the percentage value of the heat rate from the FEM simulation calculated for all possible incidence angles $(\Theta . \Phi)$,

$$
\vec{p}_{s}=\left(\begin{array}{l}
p_{1}(\Theta, \Phi) \\
p_{2}(\Theta, \Phi) \\
p_{2}(\Theta, \Phi) \\
p_{4}(\Theta, \Phi)
\end{array}\right)
$$

By resolving eq. (9) we obtain the coordinates $(\hat{\Theta}, \hat{\Phi})$, which give the minimum of the function declared in this equation - that is our wind incidence angle. This error function is superimposed in the same Fig. 18 and the minimum error is found to be for angle: $\Theta=89^{\circ}$ and $\Phi=38^{\circ}$, within an error of 7 degrees of the experimental wind direction. At that point, it is important to mention that although experiments were limited to the wind rotation angle within the horizontal plane, the wind angle retrieval algorithm was performed for full 3-D space.

So far we have not done any experiments on the sand blowing effect on the sensor performance. However, in the same tunnel we did such experiments with the REMS sensor and not observed any deviation. The fact that this spherical sensor is more robust makes us believe that similar behavior will be observed.

\section{Conclusions}

A novel spherical wind sensor anemometer based on a surface shell division into four equal spherical triangles is described. The main advantage of the spherical symmetry is that the global thermal conductance does not depend on wind incidence angle. Moreover, the simulation of the local convection heat flux at any point allow to calculate the convective heat rate of any shape of spherical sector for any incidence angle of 
the wind. Analysis shows that $9 \mathrm{~mm}$ diameter sphere divided into four triangular sectors according to the tetrahedron geometry scheme provides sensitivity of $0.2 \%$ and greater for wind velocity range $3-20 \mathrm{~m} / \mathrm{s}$. Based on the numerical simulation inverse algorithm for wind angle discrimination was proposed. The sensor components together with an assembled prototype are shown. Spherical wind sensor was successfully tested under Martian conditions. Sensor response to the different wind speed and incidence angle was in very good agreement to the numerical model of multi-physic simulation. Using wind direction discrimination algorithm we were able to retrieve satisfactorily three-dimensional wind angle coordinates $(\Theta, \Phi)$ from preliminary measurement although conditions may have changed from the ideal sphere FEM simulation model.

July 16, 2015

\section{ACKNOWLEDGMENT}

This work has been funded by the Spanish Ministry of Economy and Competitiveness under projects number: AYA2011-29967-C05-04.

\section{REFERENCES}

[1] L. Gottesdiener, "Hot wire anemometry in rarefied gas flow," Journal of Physics E: Scientific Instruments, vol. 13, no. 9, p. 908, 1980. [Online]. Available: http://stacks.iop.org/0022-3735/13/i=9/a=001

[2] H. P. Gunnlaugsson, C. Holstein-Rathlou, J. P. Merrison, S. Knak Jensen, C. F. Lange, S. E. Larsen, M. B. Madsen, P. Nrnberg, H. Bechtold, E. Hald, J. J. Iversen, P. Lange, F. Lykkegaard, F. Rander, M. Lemmon, N. Renno, P. Taylor, and P. Smith, "Telltale wind indicator for the mars phoenix lander," Journal of Geophysical Research: Planets, vol. 113, no. E3, pp. 1-6, 2008, e00A04. [Online]. Available: http://dx.doi.org/10.1029/2007JE003008

[3] C. F. Wilson, "Measurement of wind on the surface of mars," $\mathrm{Ph} . \mathrm{D}$. dissertation, Linacre College, Oxford University, 2003. [Online]. Available: http://www.researchgate.net/publication/257261894

[4] J. Merrison, H. Gunnlaugsson, J. Jensen, K. Kinch, P. Nrnberg, and $\mathrm{K}$. Rasmussen, "A miniature laser anemometer for measurement of wind speed and dust suspension on mars," Planetary and Space Science, vol. 52, no. 13, pp. 1177 - 1186, 2004. [Online]. Available: http://www.sciencedirect.com/science/article/B6V6T4DBJF3V-3/2/15a713426ac1d2c48b4a5cf6dcb664ee

[5] T. E. Chamberlain, H. L. Cole, R. G. Dutton, G. C. Greene, and J. E. Tillman, "Atmospheric measurements on mars - the viking meteorology experiment," American Meteorological Society Bulletin, vol. 57, pp. 1094-1104, Sep. 1976.

[6] S. E. Larsen, H. E. Jrgensen, L. Landberg, and J. E. Tillman, "Aspects of the atmospheric surface layers on mars and earth," Boundary-Layer Meteorology, vol. 105, no. 3, pp. 451-470, Dec. 2002. [Online]. Available: http://dx.doi.org/10.1023/A:1020338016753

[7] T. C. R. Davey and L. Harnett, "Viking meteorology instrument sensor design analysis report. document no. metc-021," TRW Systems Group, Tech. Rep., September 1973, update of the original report no. METC013.

[8] R. Sullivan, R. Greeley, M. Kraft, G. Wilson, M. Golombek, K. Herkenhoff, J. Murphy, and P. Smith, "Results of the imager for mars pathfinder windsock experiment," Journal of Geophysical Research: Planets, vol. 105, no. E10, pp. 24547-24 562, 2000. [Online]. Available: http://dx.doi.org/10.1029/1999JE001234

[9] J. T. Schofield, J. R. Barnes, D. Crisp, R. M. Haberle, S. Larsen, J. A Magalhes, J. R. Murphy, A. Seiff, and G. Wilson, "The mars pathfinder atmospheric structure investigation/meteorology (asi/met) experiment," Science, vol. 278, no. 5344, pp. 1752-1758, 1997. [Online]. Available: http://www.sciencemag.org/content/278/5344/1752.abstract

[10] J. Polkko, A.-M. Harri, T. Siili, F. Angrilli, S. Calcutt, D. Crisp, S. Larsen, J.-P. Pommereau, P. Stoppato, A. Lehto, C. Malique, and J. Tillman, "The netlander atmospheric instrument system (atmis): description and performance assessment," Planetary and Space Science, vol. 48, no. 1214, pp. 1407 - 1420, 2000, mars Exploration Program. [Online]. Available: http://www.sciencedirect.com/science/article/pii/S0032063300001197
[11] J. Murphy, G. Wilson, A. Seiff1, J. Schofield, J. Magalhae, S. Larsen6, R. Haberle, D. Crisp, and J. Barnes, "Meteorological results from the mars pathfinder lander: An overview." Lunar and Planetary Science XXIX, 1998, abstract no. 1824 .

[12] M. Dominguez, V. Jimenez, J. Ricart, L. Kowalski, J. Torres, S. Navarro, J. Romeral, and L. Castaner, "A hot film anemometer for the martian atmosphere," Planetary and Space Science, vol. 56, no. 8, pp. 1169 - 1179, 2008. [Online]. Available: http://www.sciencedirect.com/science/article/pii/S003206330800072X

[13] V. Jimenez, M. Dominguez, J. Ricart, L. Kowalski, S.Navarro, J. Torres, J.Romeral, J. Merrison, and L.Castaner, "Applications of hot film anemometry to space missions," in Eurosensors XXII, 2008.

[14] J. Gomez-Elvira, C. Armiens, L. Castaner, M. Dominguez, M. Genzer, F. Gomez, R. Haberle, A.-M. Harri, V. Jimenez, H. Kahanpaa, L. Kowalski, A. Lepinette, J. Martin, J. Martiez-Frias, I. McEwan, L. Mora, J. Moreno, S. Navarro, M. de Pablo, V. Peinado, A. Pena, J. Polkko, M. Ramos, N. Renno, J. Ricart, M. Richardson, J. RodriguezManfredi, J. Romeral, E. Sebastian, J. Serrano, M. dela Torre Juarez, J. Torres, F. Torrero, R. Urqui, L. Vazquez, T. Velasco, J. Verdasca, M.-P. Zorzano, and J. Martin-Torres, "Rems: The environmental sensor suite for the mars science laboratory rover," Space Science Reviews, vol. 170, no. 1-4, pp. 583-640, 2012. [Online]. Available: http://dx.doi.org/10.1007/s11214-012-9921-1

[15] S. Rafkin, J. Pla-Garcia, C. Newman, V. Hamilton, J. Martin-Torres, M. PazZorzano, H. Kahanpaa, and E. Sebastian, "The meteorology of gale crater determined from msl rems data and mesoscale modeling," in Eighth International Conference on Mars (2014), 2014, abstract no. 1158 .

[16] NASA-JPL, "Signs of a whirlwind in gale crater," April 2013. [Online]. Available: http://mars.jpl.nasa.gov/msl/

[17] P. Romero, G. Barderas, J. Vazquez-Poletti, and I. Llorente, "Spatial chronogram to detect phobos eclipses on mars with the metnet precursor lander," Planetary and Space Science, vol. 59, no. 13, pp. 1542 - 1550, 2011, exploring Phobos. [Online]. Available: http://www.sciencedirect.com/science/article/pii/S0032063311002091

[18] L. Kowalski, V. Jimenez, M. Dominguez-Pumar, S. Gorreta, S. Silvestre, and L. Castaner, "Low pressure spherical thermal anemometer for space missions," in SENSORS, 2013 IEEE, Nov 2013, pp. 1-4.

[19] B. van Oudheusden and A. van Herwaarden, "High-sensivity 2-d flow sensor with an etched thermal isolation structure," Sensors and Actuators A: Physical, vol. 22, no. 1, pp. 425 - 430, 1990.

[20] J. Robadey, O. Paul, and H. Baltes, "Two-dimensional integrated gas flow sensors by CMOS IC technology," Journal of Micromechanics and Microengineering, vol. 5, no. 3, pp. 243-250, 1995, cited By 43.

[21] K. A. Makinwa and J. H. Huijsing, "A smart wind sensor using thermal sigma-delta modulation techniques," Sensors and Actuators A: Physical, vol. 97-98, pp. 15 - 20, 2002, selected papers from Eurosenors $\{\mathrm{XV}\}$. [Online]. Available: http://www.sciencedirect.com/science/article/pii/S0924424702000341

[22] A. S. Cubukcu, E. Zernickel, U. Buerklin, and G. A. Urban, "A $2 \mathrm{~d}$ thermal flow sensor with sub-mw power consumption," Sensors and Actuators A: Physical, vol. 163, no. 2, pp. 449 - 456, 2010. [Online]. Available: http://www.sciencedirect.com/science/article/pii/S0924424710003705

[23] S. Sherif and R. Pletcher, "A normal sensor hot-wire/film probe method for the analysis of three-dimensional flows," Flow Measurement and Instrumentation, vol. 5, no. 3, pp. 150 - 154, 1994. [Online]. Available: http://www.sciencedirect.com/science/article/pii/0955598694900132

[24] Z.-G. Feng and E. E. Michaelides, "A numerical study on the transient heat transfer from a sphere at high reynolds and peclet numbers," International Journal of Heat and Mass Transfer, vol. 43, no. 2, pp. 219 - 229, 2000. [Online]. Available: http://www.sciencedirect.com/science/article/pii/S0017931099001337

[25] R. T. W. Sabuga, "Effect of roughness on the emissivity of the precious metals silver, gold, palladium, platinum, rhodium, and iridium," High Temperatures-High Pressures, vol. 33(3), pp. 261-269, 2001. [Online]. Available: http://www.hthpweb.com/abstract.cgi?id=htwu371

[26] J. Merrison, H. Bechtold, H. Gunnlaugsson, A. Jensen, K. Kinch, P. Nornberg, and K. Rasmussen, "An environmental simulation wind tunnel for studying aeolian transport on mars," Planetary and Space Science, vol. 56, no. 34, pp. 426 - 437, 2008. [Online]. Available: http://www.sciencedirect.com/science/article/pii/S0032063307003406

[27] S. Gorreta, E. Barajas, L. Kowalski, T. Atienza, M. Domínguez-Pumar, and V. Jiménez, "A self-calibrating closed loop circuit for configurable constant voltage thermal anemometers," Electronics Letters, September 2015. 


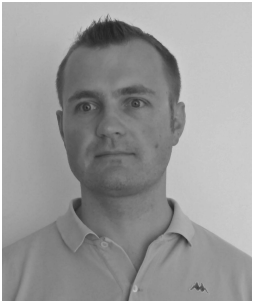

Lukasz Kowalski obtained his M.Sc. degree in the Electronics and Telecommunications from the Technical University of Lodz in 2005. He is within Universitat Politècnica de Catalunya (UPC) at Electronic Engineering Department (DEE) for his Ph.D. thesis "Contribution to advanced hot wire wind sensing". He has joined UPC Micro and Nano Technologies (MNT) Research Group in September 2005. He has participated in following space exploration projects: REMS (MSL), MEIGA (MetNet), MEDA (Mars2020). His work is aimed to the development of the thermal anemometers for the atmosphere of Mars. His research areas include dimensionless analysis, FEM/CFD simulations, thermal modeling, inverse algorithms optimization for wind speed and incidence angle retrieval In 2013, he received NASA Group Achievement Award as a member of MSL REMS Instrument Development Team, for his participation in the design of the wind sensor of the Curiosity Rover, in Gale Crater. He has coauthored two patents and several scientific papers in international journals and conferences.

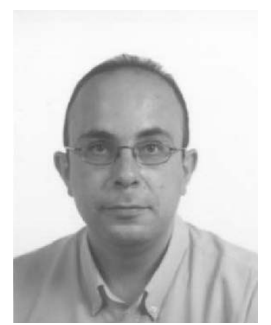

Manuel Domínguez-Pumar MSc 94, PhD97, obtained his $\mathrm{MSc}$ and $\mathrm{PhD}$ degrees in Electronic Engineering from the Universitat Politècnica de Catalunya, Barcelona, Spain, in 1994 and 1997, respectively. He obtained a Degree in Mathematics (2005) from the Universidad Nacional de Educación a Distancia (UNED), Madrid, Spain, with honours. $\mathrm{He}$ is within the UPC Department of Electronic Engineering since 1994, where he is now Associate Professor. He was visiting scholar at the Courant Institute of Mathematical Sciences from September 2006 to August 2007. He was recipient of the 2013 NASA Group Achievement Award as member of the Mars Science Laboratory REMS Instrument Development team, for his participation in the design of the wind sensor of the Curiosity Rover, in Gale Crater. He has published more than 30 research papers in peer reviewed journals. His research interests include control theory, MEMS sensors and actuators, dielectric charge control, sensors for space applications, sigma-delta modulation and dynamical systems in general.

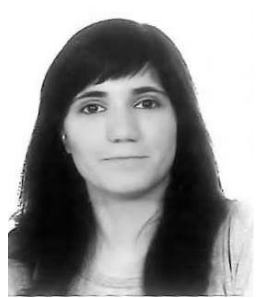

M. Teresa Atienza received the B.Sc. degree in Electronic Engineering and the M. Sc. degree in Physics Engineering from the Basque Country University, Spain, in 2012 and 2013 respectively. She joined Micro and Nano Technologies Research Group of the Universitat Politècnica de Catalunya (UPC), Spain, in October of 2013 as a Ph.D. degree student where she is working on the identification of thermal systems applied to wind sensors for space applications.

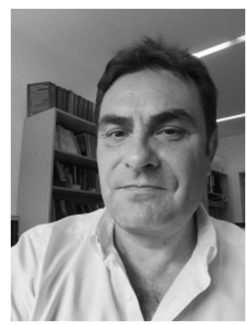

Santiago Silvestre (SM13) received the M.S. and Ph.D. degrees in telecommunication engineering from the Universitat Politècnica de Catalunya (UPC), Barcelona, Spain, in 1992 and 1996, respectively. He is currently an Associate Professor with the Departamento de Ingenieria Electrónica, Escuela Técnica Superior de Ingenieros de Telecomunicación de Barcelona, UPC. His research interests include modeling and simulation of PV systems, fault detection, and automatic supervision of PV systems.

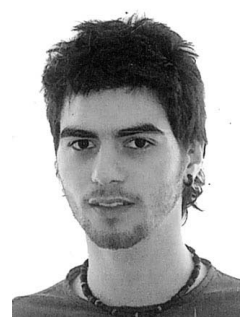

Sergi Gorreta received the M.Sc. degree in Telecomunication Engineering and the M.Sc. degree in Electronic Engineering from the Universitat Politècnica de Catalunya (UPC), Spain, in 2010 and 2011 respectively. He is currently with the Micro and Nano Technologies Research Group of the UPC as a $\mathrm{Ph} . \mathrm{D}$ degree student. His working areas are sensors for space applications, integrated circuit design, non linear circuits for MEMS and control of dielectric charging in MEMS switches.

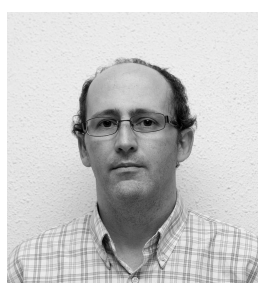

Vicente Jiménez received the M.Sc. degree in 1992 and the $\mathrm{Ph}$, degree in 1997 from the Universitat Politécnica de Catalunya (IPC), Barcelona, Spain. $\mathrm{He}$ has been with the Electronic Engineering Department, UPC, since 1992, when he became Associate Professor. His research areas include digital BiCMOS design, development of microsystem circuit interfaces, and microsystem modelling. He has participated in industry and space projects related to liquid and gas thermal flowmeters. He has coaujournals and conferences. thored more than 20 scientific papers in international

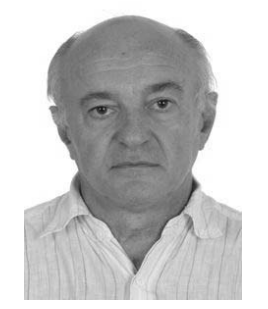

Luis M. Castañer is a Professor with the Departamento de Ingeniería Electrónica de la Escuela Técnica Superior de Ingenieros de Telecomunicación at the Universidad Politècnica de Cataluña, where he received his Doctor Ingeniero de Telecomunicación, following his undergraduate studies (Ingeniero Superior de Telecomunicación. from the Universidad Politécnica de Madrid (1971) and Diplme d'Etudes Approfondies (D.E.A.) en Physique Spatiale and Docteur-Ingenieur by the Université Paul Sabatier in Toulouse (1973)). He has also been Dean of E.T.S.I.Telecomunicación and Head of Departamento de Ingeniería Electrónica, and has held several positions in Research Agencies and Committees: representative in the Comite de Gestion y Coordinacion on non nuclear energies, DGXII Comission of the EU, Head of the Programa Nacional de Tecnologas de la Información y Comunicaciones of the CICYT (19921994), and Coordinator of the Technology Foresight at the Agencia Nacional de Evaluación y Prospectiva. He has contributed to semiconductor device research, covering solar cells in various aspects: technology of CIS, space degradation of Silicon and GaAs devices, and has contributed to the theory and technology of advanced bipolar transistors with polysilicon emitters and its application to high efficiency silicon solar cells, in particular, to the emitter resistance of these devices. He has also contributed to the design, simulation and monitoring of photovoltaic power plants and systems, and is active in the microsystems technology area, working on flow sensors, dielectric degradation of MEMS, electrostatic actuators and wind sensors for Mars. Recently he has contributed to electrowetting and electrospray techniques showing 3-D self-assembly of nanoparticles assisted by electric field. $\mathrm{He}$ is a Senior IEEE Member, a Member of the Association and Charter of Telecommunication Engineers in Spain, and a Member of the Royal Spanish Engineering Academy. 